\title{
Load Balancing Improvement in Normal and Clustered User Distribution LTE Advanced HetNets through a Hybrid Channel-Gain Access-Aware Cell Selection Scheme
}

\author{
Ajagbonna Babatunde \\ Emmanuel \\ Department of Electrical and \\ Computer Engineering, \\ Ahmadu Bello University, \\ Zaria, Kaduna State, Nigeria.
}

\author{
Abdoulie Momodou \\ Sunkary Tekanyi \\ Department of Electrical and \\ Computer Engineering, \\ Ahmadu Bello University, \\ Zaria, Kaduna State, Nigeria.
}

\author{
Mohammed Aliyu Gadam \\ Electrical Engineering \\ Department, School of \\ Engineering Technology, \\ Federal Polytechnic Bauchi.
}

\begin{abstract}
In a Macro eNodeBs (MeNBs)-Pico eNodeBs (PeNBs) deployment scenario, adopting the conventional Reference Signal Receive Power (RSRP)-based cell selection in Long Term Evolution (LTE)-Advanced Heterogeneous Networks (HetNets) causes most user equipment (UE) to connect with the MeNBs due to their higher transmit power as against that of the PeNBs, thus leading to serious load imbalance in HetNets. Therefore, this hybrid algorithm combined the channel gain-aware and the access-aware cell association metrics as a single metric for UE to base station association in LTE-Advanced HetNets deployment scenarios. The scenarios considered are the Normal distribution with uniform user distribution, which comprises of 4 PeNBs and 25 uniformly distributed UEs and the clustered distribution, which comprises of 4 PeNBs and 30 UEs, two-third of which are clustered around the the PeNBs as defined by the $3^{\text {rd }}$ Generation Partnership Project (3GPP) standard. The developed Hybrid Channel Gain Access Aware (HCGAA) scheme improved load balancing performance by $25.4 \%$ and $12.1 \%$, respectively compared with the 3GPP RSRP and RSRP +CRE cell selection. Also, an enhanced pico connection ratio of up to 1.40 times and 1.21 times that of the RSRP and RSRP +CRE cell selection schemes was achieved by the HCGAA algorithm. These improvements translate to the efficient utilization of the network resource and prevent crowding of certain cells in the network.
\end{abstract}

\section{General Terms}

Heterogeneous networks, LTE-Advanced, load balancing, channel gain and access aware cell selection algorithm

\section{Keywords}

Heterogeneous networks, LTE-Advanced, cell selection, load balancing, pico connection ratio, normal user distribution and clustered user distribution.

\section{INTRODUCTION}

Considering the unprecedented increase in the data rate demand and the large number of connected devices (which include smart phones, tablets, laptops, cameras, sensors, and smart home), it was clear that network operators needed to find sustainable ways to satisfy these constraints of diverse services [1]. For a given coverage, the deployment of a large number of small eNBs, each requiring lesser power and located close to the users, reduced the energy consumption when compared to the deployment of fewer large eNBs [2]. This necessitated the introduction of HetNets by the $3 \mathrm{GPP}$.

Consequently, to serve the increasing traffic, HetNets consisting of small cells like pico cells in macro cell layer to enhance capacity and coverage were introduced by 3GPPin order to meet the International Mobile TelecommunicationAdvanced (IMT-A) requirements for the 4G [3]. A HetNet, as shown in Figure 1, is a multilayer network comprising of different types of base stations (macro, micro, pico, and femto) that have different transmission powers and coverage areas, with the sole aim of improving the overall network energy efficiency [4]. Picocells are mostly preferred in the HetNets, because of ease of planning and deployment [5].

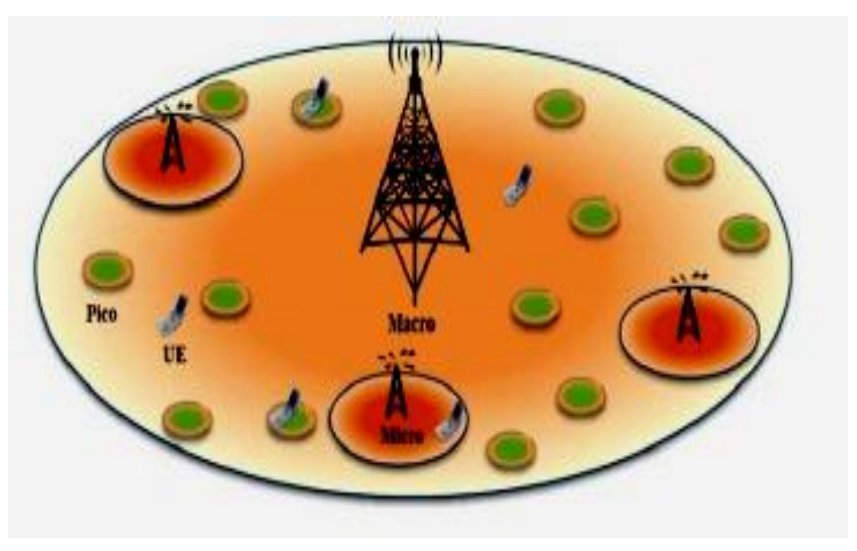

Figure 1:HetNet Deployment [4]

However, the diversity among different base stations breeds several new challenges that may significantly impact the spectral efficiency performance. For instance, during cell selection process in a HetNet, due to the diverse transmit powers of different base stations, most users prefer to associate with the MeNB with the RSRP based associations scheme. This results in the uneven distribution of traffic load among different base stations and in turn underutilization of the resources of the low power base stations [6]. Due to the low transmission power of the small eNBs, if the RSRP based cell association technique is applied in HetNet deployments, the MeNB is overloaded compared with the PeNB because 
only a few users would connect to the small cells. Thus, the available resources of the small cells would not be fully exploited while at the same time in the macro cell, the competition for the available resources would remain high [7].

In order to prevent traffic load imbalance, a fixed biased cell association scheme was developed [8]. However it lacked information on the type of resource allocation employed in the cell and also failed to detect change in traffic load condition in either PeNBs or MeNBs, and hence it could not proportionally upload the traffic load for improved system performance. In [9], the authors developed a path loss based cell association scheme that allowed UE connect to base stations that have the smallest path loss. This made more UE to connect to PeNBs due to their small radius and proximity, thus underutilization of the MeNB. The RSRP association scheme and channel access cell association metrics were combined to achieve a balanced network [6]. However, the assumption that the MeNB is always congested is not always the case, as there are situations that the PeNBs are hotspot congested. More recently, a prioritized load balancing technique for UE in a HetNet was developed based on a fixed channel gain threshold and access to nodes schemes. UE which had channel gain greater than the threshold associated with the node which gave the maximum channel gain, else its channel gain was combined with the access probability to the node [10]. However, the simulation scenario did not represent a realistic UE distribution and the threshold selection criterion was not stated.

In this paper, the load imbalance problem in a HetNet is solved by combining a user to base station association algorithm that is based on the channel gain between the UE and the base stations, and the access probability to base stations. The access-aware (access probability) was formulated as the reciprocal of the number of UEs in a cell to prevent UE from connecting to cells that are overcrowded. Thus, cell association was derived as a function of the numbers of UEs present in a cell. Then, extensive simulations were performed using MATLAB 2013a for both normal UE distribution and clustered UE distribution scenarios and the results show that the developed HCGAA algorithm performs better than the RSRP and RSRP+CRE based cell association in terms of load balancing fairness and pico connection ratio.

The remainder of this paper is organized as follows. In Section 2, the background to the various cell association schemes is presented. Section 3 presents the system model and basic metrics. Implementation of the developed algorithm is presented in section 4. Section 5 gives simulation result and discussions and the whole paper is concluded in Section 6, with some suggestions for future works, and references used are captured in Section 7.

\section{BACKGROUND}

Cell selection entails the procedure through which UE attach to either PeNB or MeNB using the received signal strength assigned to resources for traffic offloading. Cell selection can thus achieve traffic load balancing and throughput maximization of the UE by using the available resource allocated [11]. Some major cell selection schemes are:

\subsection{Maximum Reference Signal Received Power based Cell Association}

The RSRP from base stations is used to determine the appropriate base station to associate UE with in a network. At the time of cell selection, UE gets associated with the base station providing the highest RSRP. So, the $i^{\text {th }}$ UE selects the $k^{\text {th }}$ base station as its serving base station if,

$$
\operatorname{CellID}_{i}=\arg _{k} \max \left(R S R P_{k}\right)
$$

In a HetNet, the maximum RSRP scheme makes UE always get associated with base station providing highest SINR, as such, most UE prefer to associate with the high power base stations because of its greater transmit power as seen in Figure 2 [12]. This results in the uneven distribution of traffic load among different base stations and in turn underutilization of the resources at low power base stations [13].

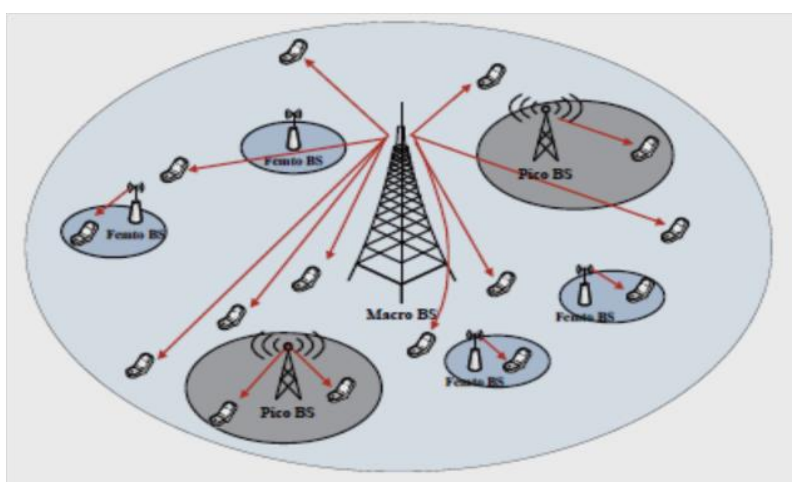

Figure 2: RSRP based Cell Association of a Three-Tier HetNet [12].

\subsection{Cell Range Expansion based Association}

The coverage area of a PeNB and the association opportunity for UE to connect to the PeNB are increased by adding a positive bias value to the RSRP from PeNBs [14]:

$$
\text { Cell ID } D_{i}=\arg _{k} \max \left(R S R P_{k}+\operatorname{bias}_{k}\right)(2)
$$

where, bias is zero for the macro cell and has a non-negative value for the small cells, resulting in more users association with the smaller cells.

\section{SYSTEM MODEL AND SIMULATION SET UP}

The considered simulation environment in this paper is based on the 3GPP LTE system level simulations toolbox, having 19 hexagonal wrap around cells (57 MeNBs). Each of the cells has three sectors with each sector considered an active LTE eNodeB having 4 PeNB per sector. The PeNBs are randomly dropped within the sector with minimum inter-site distance constrains. A hexagonal cell has 3 directional antennas at 120 degrees apart, one for each MeNB sector and the PeNB has one omni-directional antenna. Users are assumed to be uniformly distributed throughout the coverage area normal UE distribution scenario and a fraction of users is clustered around the PeNB in the clustered UE distribution scenario as seen in Figure 3 and Figure 4, respectively. Mobility is represented by users having different locations in each drop. 


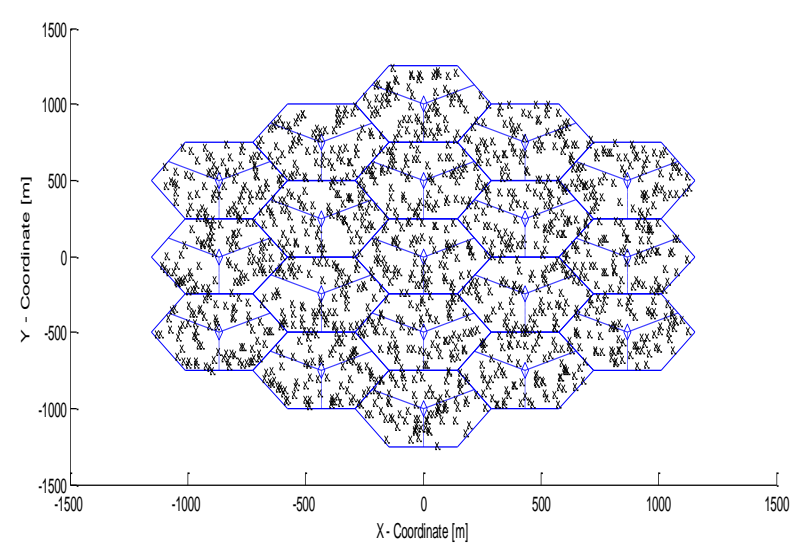

Figure 3: Schematic Diagram of Normal UE Distribution Scenario [15]

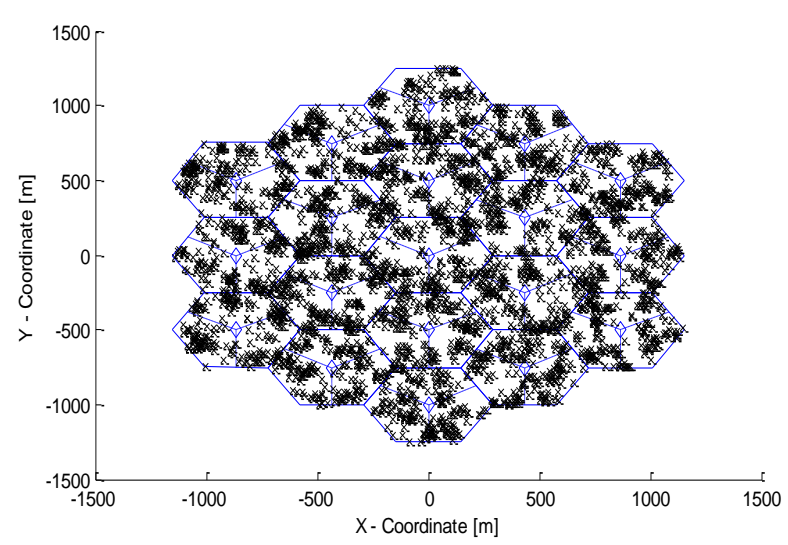

Figure 4: Schematic Diagram of Clustered UE Distribution Scenario [15]

\subsection{Load Balance Model}

Load balancing belongs to the group of suggested selforganising network functions for LTE network operations, which is meant to deliver extra gain in terms of network performance. Load balancing is achieved by adjusting the network control parameters in such a way that overloaded cells can offload the excess traffic to low-loaded adjacent cells whenever available [16].

Jain's Fairness index is employed to measure the status of the system's load balance. It is a load balance index measuring the degree of load balancing of the entire network and it is given as follows [10]:

$$
\rho(t) \frac{\left(\sum \sigma_{k(t)}\right)^{2}}{|M| \Sigma\left(\sigma_{k(t)}\right)^{2}}
$$

where, $\rho(t)$ is the fairness in the load balance, $\sigma_{k(t)}$ represent the load in any cell $\mathrm{k}$, and $M$ represent the total number of MeNB and PeNB in the system.

\subsection{Pico Connection Ratio}

The pico connection ratio is the number of UE connected to the PeNBs divided by the total number of UE in the network, after the selection process. If after cell selection process, the number of UEs connected to pico cells is $n_{p c}$ and the total number of UEs in that HetNet is $N_{\text {het }}$, therefore the pico connection ratio is given as:

$$
P_{C R}=n_{p c} / N_{\text {het }}
$$

where, $P_{C R}$ represent the pico connection ratio.

\section{HYBRID CHANNEL-GAIN ACCESS- AWARE CELL SELECTION SCHEME}

The hybrid algorithm is a combination of the channel gain cell selection algorithm and access aware cell association algorithm. An access probability which is the inverse of the number of user in a cell when round robin scheduling is adopted is combined with the channel gain and used as the association metric to prevent the overload of an eNB and also to ensure full utilization of the resource of the eNBs. The channel gain between an eNB and a UE in the downlink is given asthe difference between the receive power and the transmit power. The power is transmitted from the base station to the UE. These powers are mathematically related as [17]:

$$
G_{P}=P_{R}-P_{T}
$$

where, $G_{P}$ is the channel gain, $P_{R}$ is the receive power at the $\mathrm{UE}$ and $P_{T}$ is the transmit power at the base station. When $G_{P}$ is negative, it signifies a loss.

In a large scale fading environment, all that the transmit signal passes through before getting to the UE constitutes the channel gain which is given as [18]:

$$
G_{P}=-P L+S F+A G-L m i s c
$$

where, $P L$ represent the path loss between the base station and the UE, $S F$ is shadow fading, $A G$ represent antenna gain, Lmisc is any residual miscellaneous loss such as attenuation in the RF feeder cables or outdoor to in-car loss.

The path loss and shadow fading vary with carrier frequency and distance, whether the UE is indoor or outdoor and whether the UE link is in line of sight or non-line of sight. Path loss and shadow fading are known as slow fading [18].

Path loss is the dissipation of the transmit power as it travels towards the receiver. Path loss is also as a result of the effect of the propagation channel, while shadowing occurs as a result of the various obstacles the transmit signal encounters due to absorption, scattering, diffraction, and reflection.

The access probability using round robin resource allocation for UE connecting to MeNB and PeNB is respectively given as [6]:

$$
\begin{aligned}
p_{m}^{A} & =\left(\frac{1}{N_{m}+1}\right) \\
p_{p}^{A} & =\left(\frac{1}{N_{p}+1}\right)
\end{aligned}
$$

where, $p_{m}^{A}$ and $p_{p}^{A}$ are the probability of UE associating with the MeNB and PeNB, respectively and $N_{m}$ and $N_{p}$ are the number of UE in the MeNB and PeNB, respectively.

In HetNets, the same spectrum is reused in MeNBs and PeNBs and when round robin scheduling is employed, equal time resources are assigned to each UE, hence, the load in a cell can be represented by the number of UE in that cell [19].

The hybrid cell selection algorithm metric for UE to connect to the MeNBs and PeNBs are respectively given as:

$$
\begin{aligned}
& \text { Hybrid }_{m}=G_{P} \times\left(\frac{1}{N_{m}+1}\right) \\
& \text { Hybrid }_{p}=G_{P} \times\left(\frac{1}{N_{p}+1}\right)
\end{aligned}
$$

Therefore if Hybrid $_{p}$ is greater than Hybrid $_{m}$, the UE is connected to the PeNB, else it is connected to the MeNB. The 
input to the algorithm is acquired from the network side. The algorithm is implemented in the MeNB and executed through the $\mathrm{X}-2$ interface.

\subsection{Steps of the Hybrid Channel-Gain Access-Aware (HCGAA) Cell Selection Algorithm}

The steps taken to implement the HCGAA cell selection algorithm are:

Step 1: Initialization of parameters: Configure simulator input, generate MeNB topology, generate pico topology, and generate UE topology.

Step 2: Compute channel gain between UE an eNB using (5).

Step 3: Compute pico and macro access probability using equation (7) and (8) respectively.

Step 4: Compute Hybrid H $_{m}$ and Hybrid ${ }_{p}$ using (9) and (10) respectively.

Step 5: If Hybrid $_{p}$ is greater than Hybrid ${ }_{m}$, connect UE to PeNB else connect UE to MeNB.

Step 6: Compute Jain's fairness load balancing index using (3)

Step 7: Compute pico connection ration using (4)

\section{SIMULATION RESULT AND DISCUSSIONS}

In the following, we compare the performance of the conventional RSRP, RSRP + CRE bias of $4 \mathrm{~dB}$, channel gain, and the developed HCGAA cell selection algorithm for normal UE distribution and clustered UE distribution scenarios. For the RSRP + CRE, a $4 \mathrm{~dB}$ bias is used in order not to incur serious interference. Load balance fairness and pico connection ratio were used to evaluate the performance of the algorithms.

\subsection{Load Balancing Fairness}

The Jain fairness load balance index for the RSRP, RSRP+CRE, Channel gain and HCGAA algorithms were computed and plotted for normal UE distribution and clustered UE distribution scenarios. This metric value was computed after the cell association process. The graph of the Jain fairness load balance index with $4 \mathrm{PeNB}$ and increasing UE for the normal UE distribution scenario is depicted in Figure 5. With 45 users as shown, the HCGAA load balancing performance is $25.4 \%$ and $12.1 \%$ better than that of the RSPR and RSRP +CRE schemes, respectively. It is observed that the load balancing index increases as the number of users increases for the HCGAA and channel gain aware cell selection scheme, while for the RSRP and RSRP + CRE, as the numbers of users increases the load balancing performance drops. This indicates that the HCGAA scheme is better in terms of load balancing and can be implemented in dense populated areas.

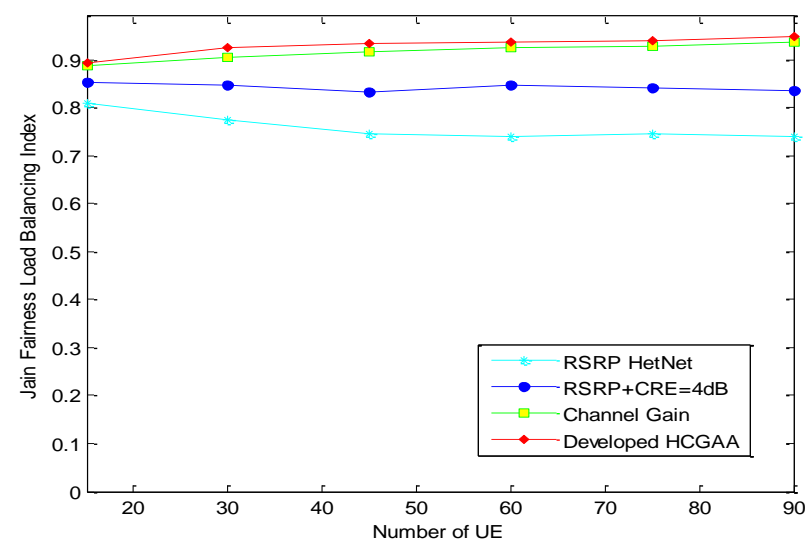

Figure 5: Jain Load Balancing Index with 4 PeNBs and Increasing UE for Normal UE Distribution Scenario

For the clustered UE distribution scenarios, it is observed that the load balancing index of the HCGAA and RSRP +CRE schemes show a slight identical load balancing performance. With 4 PeNBs and 45 users as shown in Figure 6, the improvement of the HCGAA cell selection scheme over that of the RSPR and RSRP +CRE schemes is by $5.3 \%$ and $1.5 \%$, respectively. The HCGAA and RSRP +CRE schemes show a slight identical load balancing performance.

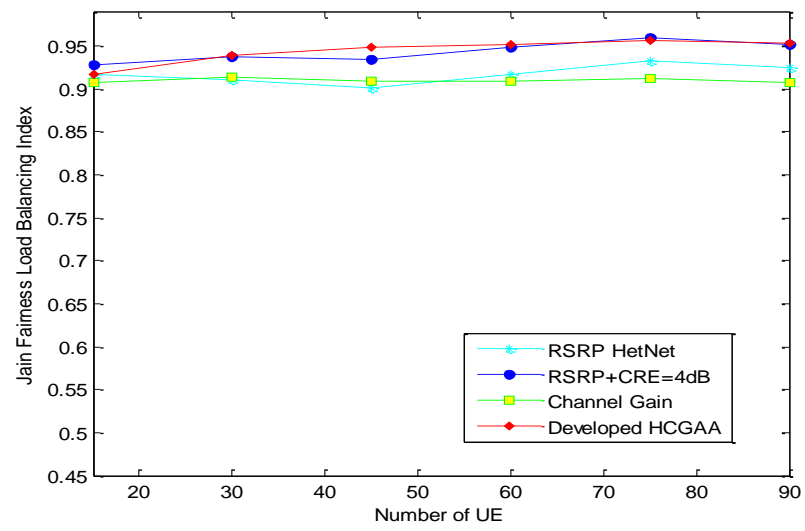

Figure 6: Jain Load Balancing Index with 4 PeNBs and Increasing UE for Clustered UE Distribution Scenario

The graphs of the Jain fairness load balancing index with fixed number of UE and increasing PeNBs for the normal UE distribution and clustered UE distribution scenarios are depicted in Figure 7 and Figure 8, respectively.

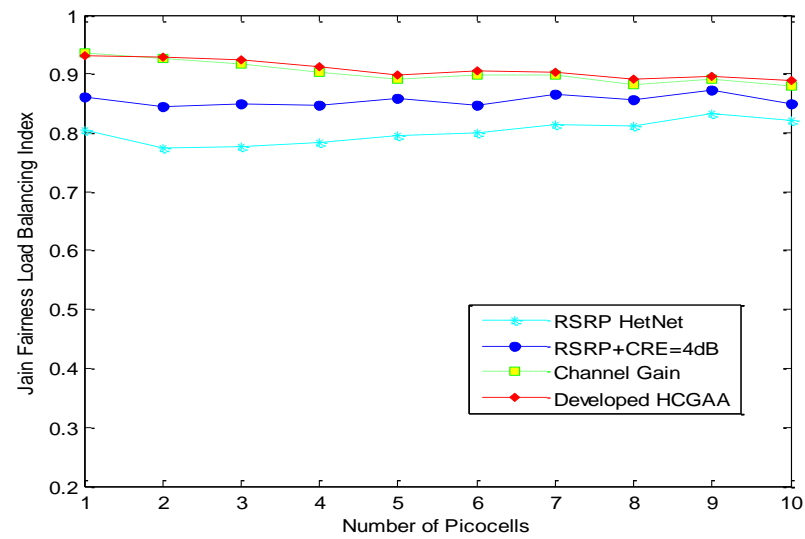

Figure 7: Jain Load Balancing Index with 25 UE and Increasing PeNBs for Normal UE Distribution Scenario 


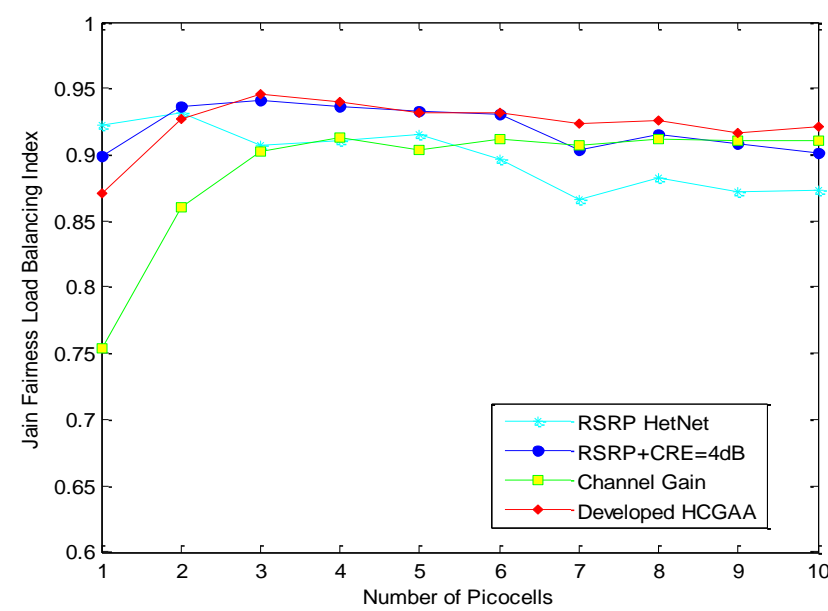

Figure 8: Jain Load Balancing Index with 30 UE and Increasing PeNBs for Clustered UE Distribution Scenario

From Figure 7, with 25 users, as the number of PeNB increases, the load balancing performances of all the schemes slightly drop for the normal UE distribution scenario. However, for the clustered UE distribution scenario, the load balancing index of the schemes starts depreciating when the number of PeNB is 3 for the HCGAA and RSRP + CRE schemes and 2 for the RSRP scheme as depicted in Figure 8. For the normal UE distribution scenario, with 7 PeNBs and 25 UE as shown in Figure 7, the load balancing performance of the HCGAA is $10.7 \%$ and $4.2 \%$ better than the RSPR and RSRP +CRE schemes, respectively. Also, for the clustered UE distribution scenario, the improvement over the RSPR and RSRP +CRE schemes is by $6.7 \%$ and $2.1 \%$, respectively. These results show that a hybrid cell selection scheme that utilizes the channel gain metric and the access probability of connecting to a base station together as the cell association initiator, places the users more evenly than other association schemes. Thus, with the HCGAA scheme, cells are not over loaded or underutilized as in the RSRP and RSRP +CRE schemes.

\subsection{Pico Connection Ratio}

The pico connection ratio was computed for the various schemes. It is the number of UE connected to the PeNBs divided by the total number of UE in the network, after the selection process. The graphs of the pico connection ratio with 4 PeNB and increasing UE for the normal UE distribution and the clustered UE distribution scenarios are depicted in Figure 10 and Figure 11, respectively

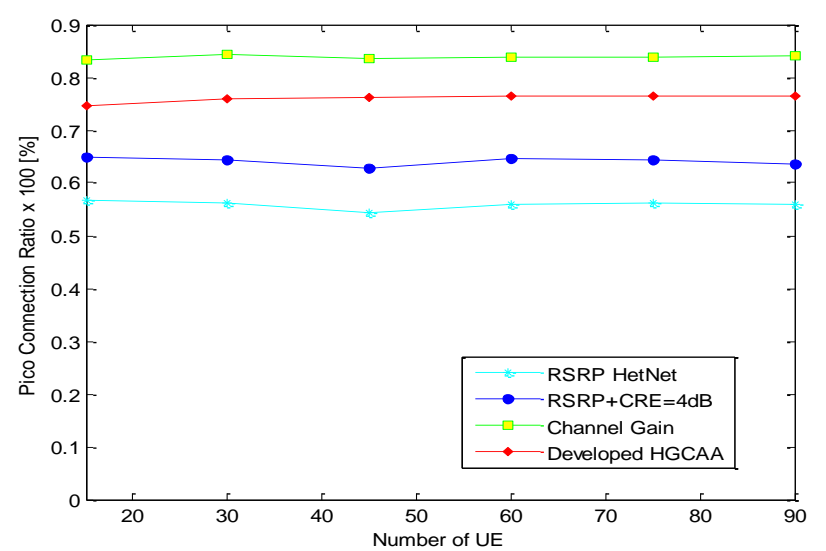

Figure 9: Pico Connection Ratio for 4 PeNBs with Increasing UE for Normal UE Distribution Scenario

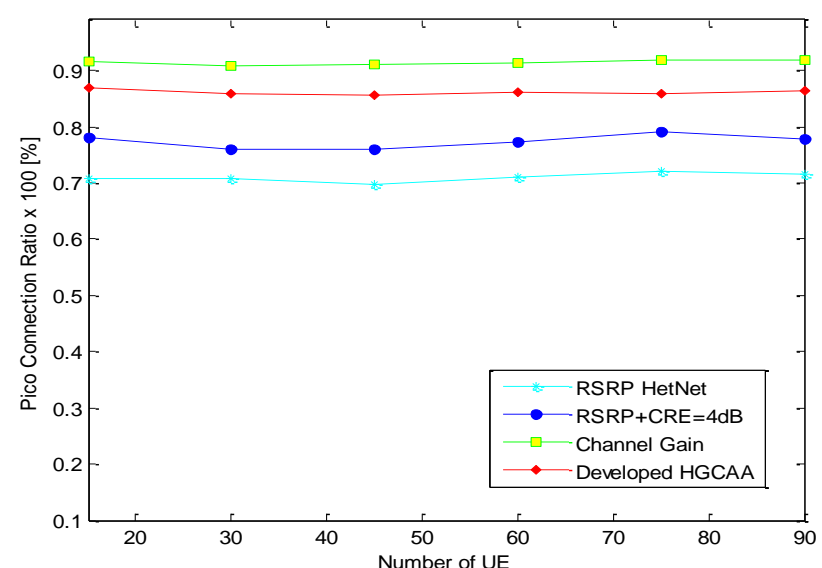

Figure 10: Pico Connection Ratio for 4 PeNBs with Increasing UE for Clustered UE Distribution Scenario

For the normal UE distribution scenario and the clustered UE distribution scenario, with 4 PeNBs, the pico connection ratios of the various cell association schemes remained fairly stable as the number of UE increases, as shown in Figure 9 and Figure 10, respectively. However, the pico connection ratio is highest for the channel gain cell selection scheme for both scenarios because the UEs are generally closer to the PeNB than the MeNB. For the normal UE distribution scenario, with 4 PeNBs and $45 \mathrm{UE}$, the pico connection ratio of the channel gain cell selection scheme is 1.54 times and 1.33 times more than that of the RSRP and RSRP +CRE schemes, respectively, while pico connection ratio of the HCGAA scheme is 1.40 times and 1.21 times more than that of the RSRP and RSRP +CRE scheme, respectively. For the clustered UE distribution scenario, also with 4 PeNBs and 45 UE, the pico connection ratio of the channel gain cell selection scheme is 1.30 times and 1.2 times more than that of the RSRP and RSRP +CRE schemes, respectively, while the pico connection ratio of the HCGAA scheme is 1.23 times and 1.13 times more than that the RSRP and RSRP +CRE schemes, respectively.

The graphs of the pico connection ratio for fixed number of UE and increasing PeNBs for the normal UE distribution and the clustered UE distribution scenarios are depicted in Figure 11 and Figure 12, respectively.

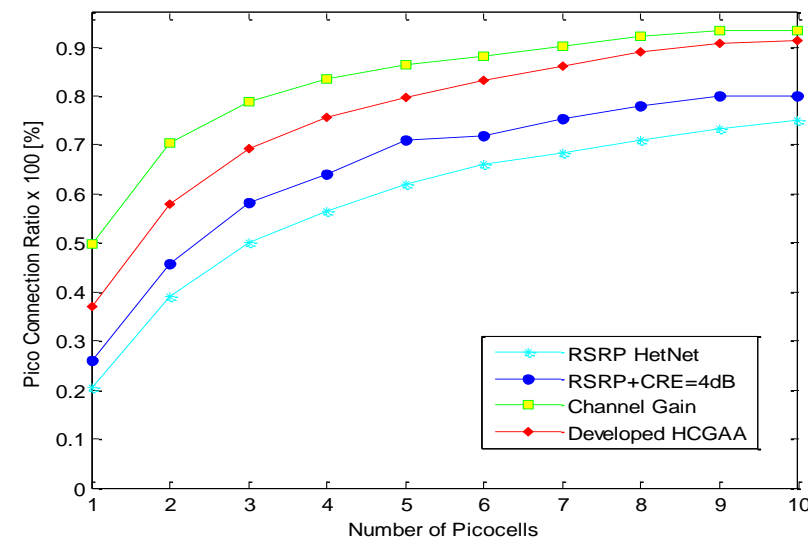

Figure 11: Pico Connection Ratio for 25 UE with Increasing PeNBs for Normal UE Distribution Scenario 


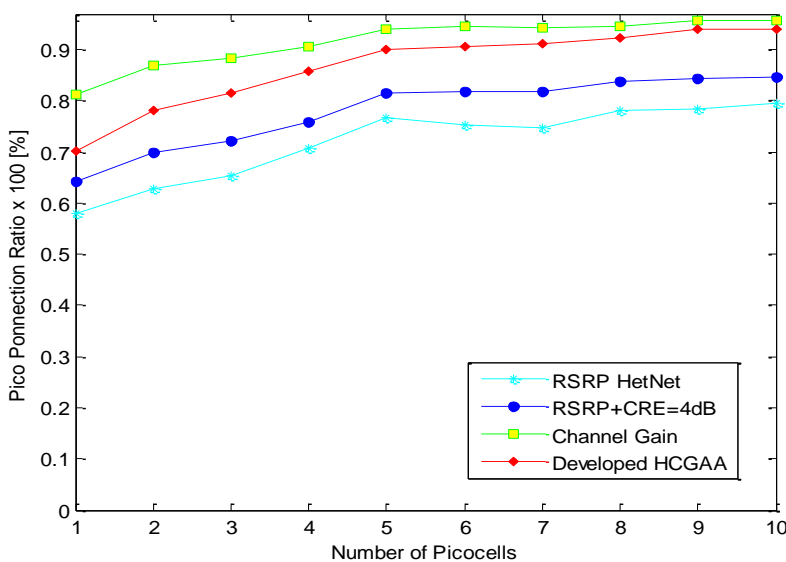

Figure 12: Pico Connection Ratio for $30 \mathrm{UE}$ with Increasing PeNBs for Clustered UE Distribution Scenario

When the number of UE is fixed, it is discovered from Figure 11 and Figure 12 that the pico connection ratios of the cell selection schemes increase as the number of PeNBs increases for the normal UE distribution and clustered UE distribution scenarios. For the normal UE distribution scenario, with 7 PeNBs and $25 \mathrm{UE}$, the pico connection ratio of the channel gain cell selection scheme is 1.32 times and 1.20 times that of the RSRP and RSRP +CRE cell selection schemes, respectively, while the pico connection ratio of the HCGAA scheme is 1.26 times and 1.15 times that of the RSRP and RSRP +CRE cell selection schemes, respectively. For the clustered UE distribution scenario, and with 7 PeNBs and 30 $\mathrm{UE}$, the pico connection ratio of the channel gain cell selection scheme is 1.26 times and 1.15 times that of the RSRP and RSRP +CRE cell selection schemes, respectively, while the pico connection ratio of the HCGAA scheme is 1.22 times and 1.12 times that of the RSRP and RSRP +CRE cell selection schemes, respectively.

It is observed from Figure 11 and Figure 12 that the pico connection ratio of the channel gain aware scheme is higher than that of the HCGAA cell selection scheme, this is due to the proximity of pico cells to the UEs, thus utilising only the channel impediments as the association criteria causes more UEs to connect to the pico cells. The data obtained show how the channel gain and HCGAA scheme offload more traffic to the PeNBs after the cell selection process compared with the RSRP and RSRP+CRE cell selection schemes.

\section{CONCLUSION AND FUTURE WORKS}

In a multi-tier Hetnet deployment comprising MeNBs and PeNBs, cell association suffers great unbalanced traffic load problem. The diverse transmission power of the MeNBs and PeNBs makes the conventional RSRP based cell association not viable because it causes more UE to tend to connect to the MeNBs thereby causing traffic load imbalance. The unbalanced load situation results in the underutilization of the available resources of the PeNBs. In this research paper, we developed and implemented a hybrid algorithm to improve load balancing in a normal UE distribution and clustered UE distribution LTE-Advanced HetNet by combining the channel gain association and access probability of connecting to a cell as a single metric for UE to cell association. The developed HCGAA scheme improved load balancing fairness and pico connection ratio as compared to the standard 3GPP RSRP and RESP +CRE cell selection algorithm. It would be interesting to study the impact of interference on load balancing and energy efficiency of the HCGAA scheme. Other cell selection algorithms based on data rates and distance aware could be employed in cell selection criteria.

\section{REFERENCES}

[1] Davaslioglu, K. (2015). Energy Efficiency and Load Balancing in Next-Generation Wireless Cellular Networks Dissertation. University Of California, Irvine.

[2] Fettweis, G., \& Zimmermann, E. (2008). ICT energy consumption-trends and challenges. Paper presented at the Proceedings of the 11th International Symposium on Wireless Personal Multimedia Communications.

[3] Tong, E., Ding, F., Pan, Z., \& You, X. (2015). An energy minimization algorithm based on distributed dynamic clustering for long term evolution (LTE) heterogeneous networks. Science China Information Sciences, 58(4), 112.

[4] Abdulkafi, A. A., Tiong, S. K., Chieng, D., Ting, A., Ghaleb, A. M., \& Koh.J. (2013). Modeling of Energy Efficiency in Heterogeneous Network. Paper presented at the Research Journal of Applied Sciences, Engineering and Technology, Maxwell Scientific Organization.

[5] Guo, W., Turyagyenda, C., Hamdoun, H., Wang, S., Loskot, P., \& O'Farrell, T. (2011). Towards a low energy LTE cellular network: Architectures. Paper presented at the Signal Processing Conference, 2011 19th European.

[6] Siddique, U., Tabassum, H., \& Hossain, E. (2015). Channel Access-Aware User Association with Interference Coordination in Two-Tier Downlink Cellular Networks.

[7] Tian, P., Tian, H., Zhu, J., Chen, L., \& She, X. (2011). An adaptive bias configuration strategy for range extension in LTE-advanced heterogeneous networks. Paper presented at the Communication Technology and Application (ICCTA 2011), IET International Conference on.

[8] Damnjanovic, A., Montojo, J., Wei, Y., Ji, T., Luo, T., Vajapeyam, M., Yoo, T., Song, O., and Malladi,D.,"A survey on 3GPP heterogeneous networks," IEEE Wirel. Commun., vol. 18, pp. 10-21, May 2011.

[9] Wu, J., Rangan, S., \& Zhang, H. (2012). Green Communications: Theoretical Fundamentals, Algorithms and Applications: CRC Press.

[10] Gadam, M., Ng, C., Nordin, N., Sali, A., \& Hashim, F. (2016). Hybrid channel gain prioritized access-aware cell association with interference mitigation in LTE-Advanced HetNets. International Journal of Communication Systems.

[11] Salman, M. I., Abdulhasan, M. Q., Ng, C. K., Noordin, N. K., Sali, A., \& Mohd Ali, B. (2013). Radio resource management for green $3 \mathrm{gpp}$ long term evolution cellular networks: review and trade-offs. IETE Technical Review, 30(3), 257-269.

[12] Ye, Q., Rong, B., Chen, Y., Al-Shalash, M., Caramanis, C., \& Andrews, J. G. (2013b). User association for load balancing in heterogeneous cellular networks. Wireless Communications, IEEE Transactions on, 12(6), 27062716. 
[13] Darshna Prajapati, \& Richhariya, V. (2014). A Survey on Cell Selection Schemes for Femtocell Networks. International Journal of Engineering Research, 3(7), 465-468.

[14] Sun, S.-S., Liao, W., \& Chen, W.-T. (2014). Traffic offloading with rate-based cell range expansion offsets in heterogeneous networks. Paper presented at the Wireless Communications and Networking Conference (WCNC), 2014 IEEE.

[15] Tombaz, S., Usman, M., \& Zander, J. (2011). Energy efficiency improvements through heterogeneous networks in diverse traffic distribution scenarios. Paper presented at the Communications and Networking in China (CHINACOM), 2011 6th International ICST Conference on.
[16] Adeyemi, A. S., \& Ike, D. U. (2013). A Review of Load Balancing Techniques in 3GPP LTE System. Int. J. Comput. Sci. Eng, 2(4), 112-116.

[17] http://www.3gpp.org/Specificationcans-groups/, 9th September, 2015

[18] Ericsson, (2009) "R1-091320 Radio characteristics of the ITU test environments and deployment scenarios," $3 G P P$ TSG RAN WG 1, Meeting 56bis, March 2009.

[19] Capozzi, F., Piro, G., Grieco, L. A., Boggia, G., and Camarda, P.,"Downlink packet scheduling in LTEcellular networks: Key design issues and a survey," IEEE Commun. Surv. Tutorials, vol. 15, no. 2, pp.678700, 2013. 